\title{
Integrated Field, Laboratory, and Modeling Studies to Determine the Effects of Linked Microbial and Physical Spatial Heterogeneity on Engineered Vadose Zone Bioremediation
}

(Project Number: 70165)

\section{Principal Investigator}

Fred Brockman

Pacific Northwest National Laboratory

P.O. Box 999, MSIN P7-50

Richland, WA 99352

509-376-1252 (phone)

fred.brockman@pnl.gov

\section{Co-Investigator}

John Selker

Oregon State University

Gilman Hall 240

Corvallis, OR 97330

541-737-6304 (phone)

selkerj@engr.osu.edu

\section{Graduate Students and Research Assistants}

Steven Bradley, post-doctoral

Mark Rockhold, post-doctoral

Rockie Yarwood, post-doctoral

Tina Spadoni, B.S., research assistant

Michael Niemet, M.S., research assistant

Nisha Kapadia, B.S.

Thomas Perry, B.S. 


\section{Research Objective}

In situ bioremediation of contaminants can offer advantages in cost, speed, public acceptance, and final cleanup levels achieved relative to physical removal methods. However, microbial populations in the unsaturated zone are spatially discontinuous and sparse, especially in deep vadose zones and in arid climates with very low moisture and nutrient flux. In addition, there is a lack of knowledge on 1) the ability of microbes to colonize "empty" regions of the vadose zone in response to nutrient delivery and 2) how microbial colonization is controlled by hydrologic and physical features. These issues raise questions about the feasibility of deep vadose zone bioremediation and the accuracy of flow and transport models for vadose zone bioremediation.

The goal of this research is to provide DOE with an increased understanding of the effect of interacting hydrologic and microbiological processes that control the feasibility of engineered bioremediation of chlorinated compounds in heterogeneous, microbially sparse deep vadose zones. The specific objectives are

- to conduct laboratory research on vadose zone microbial colonization processes as a function of hydrologic and physical features and use the information to develop an improved vadose zone reactive transport model

- to evaluate a gas-phase nutrient delivery approach for enhancing removal of carbon tetrachloride from the vadose zone.

\section{Research Progress and Implications}

This report summarizes the progress achieved during the first 20 months of a 3-year project. Field sampling at the Hanford Site 216-Z-9 trench (contaminated with carbon tetrachloride and transuranic radionuclides), originally scheduled for the beginning of the project (fall/winter 1999), was delayed until late May and early June 2001. Twenty-four core samples ranging in depth from 102 to $187 \mathrm{ft}$ below ground surface are being collected. The field sampling is to determine 1) what types of microbial metabolisms exist throughout the depth profile, 2) the ability of indigenous microorganisms to grow using gaseous sources of nitrogen, phosphorus, and carbon, and 3) the ability of indigenous microorganisms to degrade carbon tetrachloride.

In the interim, research has focused on unsaturated model porous media systems. Research tasks under way at Pacific Northwest National Laboratory are addressing the ability of microbes to colonize uninhabited porous media under static unsaturated conditions. At Oregon State University, researchers are examining the dynamics of microbial metabolic and colonization processes under flowing unsaturated conditions. Both efforts involve understanding how microbial colonization is controlled by porous media water content and particle size.

Microbial motility - the ability of microorganisms to swim through water or move over surfaces - has been largely discounted as unimportant in microbial colonization of the subsurface. However, no specific studies of microbial motility under vadose zone conditions are 
reported in the literature. Our research shows that motile bacteria can colonize rapidly in static (no flow) unsaturated porous media. This adds to the existing base of scientific knowledge on phenomena under unsaturated conditions and challenges conventional scientific wisdom.

Specifically, in 1-mm-diameter sand, two species of Pseudomonas bacteria (one that degrades carbon tetrachloride) migrated upward in columns at initial rates of $5 \mathrm{~cm} / \mathrm{h}$ in porous media columns at a calculated average water film thickness of $70 \mu \mathrm{m}$ (equivalent to $20 \%$ volumetric water content, $\mathrm{vWC}$ ). Initial migration rates were 0.03 to $0.07 \mathrm{~cm} / \mathrm{h}$ at a calculated average water film thickness of $18 \mu \mathrm{m}$ (equivalent to $5 \% \mathrm{vWC}$ ). These rates were achieved by (previously well-fed) cells in the absence of nutrients in the column, as well as by cells in the presence of nutrients in the column. Migration ceased after several days in the absence of nutrients in the column. Migration was not detected at a calculated average water film thickness of $4 \mu \mathrm{m}$ (equivalent to $1.3 \% \mathrm{vWC}$ ) regardless of whether nutrients were absent or present in the column, demonstrating that very low water contents prevent motility-driven colonization (Figure 1). The significance of these results is that nutrient delivery to coarse-grained regions of the vadose zone could promote colonization by motile bacteria. If the motile bacteria were able to degrade contaminants, colonization of previously "empty" regions would dramatically increase the rate and efficiency of biodegradation.

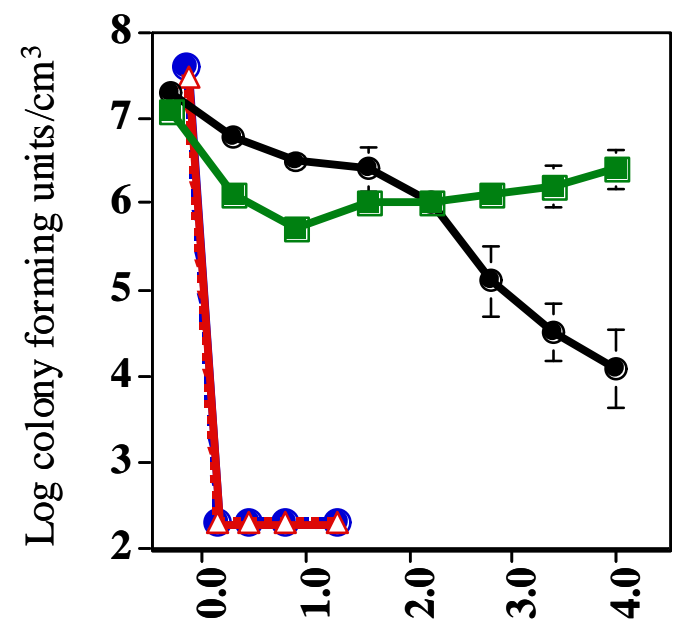

Figure 1. Effect of Volumetric Water Content on Motility-Driven Migration of Ps. Stutzeri KC in Static Unsaturated Columns. Bacteria were inoculated at -0.6 to $0.0 \mathrm{~cm}$. Three of the four treatments did not contain nutrients to support growth; the treatment designated with triangles contained nutrients.

Migration distance (cm)

The ability of microbes to colonize unsaturated porous media in the presence of flow and soluble nutrients was studied in a two-dimensional chamber, $40 \mathrm{~cm}$ wide by $60 \mathrm{~cm}$ high by $1 \mathrm{~cm}$ thick, instrumented to allow periodic visualization of water distribution, nutrient delivery, and microbial activity. Water distribution is visualized by light transmission, nutrient delivery by use of a dye, and microbial activity by periodic addition of salicylate, which causes the genetically engineered bacterium Pseudomonas fluorescens HK44 to produce light in the presence of oxygen. A charge-coupled device camera records data at high resolution $(1 \mathrm{~mm} / \mathrm{pixel})$ and quantifies, in situ, the temporal and spatial interactions between water content, solute transport, and microbial processes. Experiments were conducted with homogenous porous media and with heterogeneous porous media consisting of a wedge of coarse sand within a matrix of finer sand. 
Homogeneous porous media experiments were funded largely by a previous National Science Foundation project, with EMSP funds used for final analyses and numerical modeling. EMSP funds also served to extend the homogeneous systems to heterogeneous layered systems, which are more typical of Hanford sediments.

In a representative homogeneous experiment, microbial colonization spread from a 4-cmdiameter inoculated region to more than $17 \mathrm{~cm}$ in width in 6 days (Figure 2). Colonization advanced upward approximately $3 \mathrm{~cm}$ against a pore water velocity of $60 \mathrm{~cm} / \mathrm{h}$. By Day 3 , the interior region of the colonized zone no longer responded to the application of salicylate, indicating that oxygen was depleted in this region. Microbial growth caused a 5 -cm lowering of the capillary fringe in the chamber and a localized zone of desaturation in the vicinity of the most heavily colonized region. The permeability of the most heavily colonized area was reduced enough to alter water flow significantly.

Experiments with heterogeneous porous media employed a wedge of coarse sand to provide a capillary barrier and introduce initial spatially variable flow. Processes similar to those noted in the homogeneous experiments were observed (Figure 3). In addition, microbial growth caused an increase in the water content perched upon the wedge. Although the increase in water content was insufficient for flow to break through the capillary barrier into the fine sand under the conditions of the one-week experiment, we believe longer experiments or a slightly higher applied flux would produce this result.

These experiments demonstrate that microbial growth causes dynamic changes in flow paths and hydraulic properties. They also indicate that physical heterogeneity strongly controls microbial activity and colonization in the unsaturated zone.

Results from these experiments are being used as input to parameterization and testing of a twodimensional finite-difference numerical model for predicting contaminant fate and transport in the vadose zone. This model accounts for water flow, transport of solutes and bacteria, microbial growth and degradation kinetics, gas diffusion, and interphase exchange. The model captures new information on interactions between microbial dynamics and vadose zone processes that can be applied in conjunction with experimental studies to gain insights into, and greater understanding of, these processes and phenomena.

We also have collaborated with the U.S. Geological Survey on its Toxic Substances Hydrology Program in the analysis of samples from the Amargosa Desert Research Site (ADRS) in Beattie, Nevada, to determine if far-field migration of ${ }^{14} \mathrm{C}-\mathrm{CO}_{2}$ may be attributable to microbial activity. Land disposal of low-level mixed organic-radioactive waste occurred at both the ADRS and the Hanford Site, and the ADRS serves as an analog for understanding processes occurring at Hanford. The data showed that microbial populations and activity were very low away from the burial trenches and the capillary fringe and would not be generating measurable ${ }^{14} \mathrm{C}-\mathrm{CO}_{2}$. The results indicate that microorganisms in and immediately adjacent to the buried waste are generating the ${ }^{14} \mathrm{C}-\mathrm{CO}_{2}$ and that physical transport processes controlled by the site geology are causing far-field migration. 

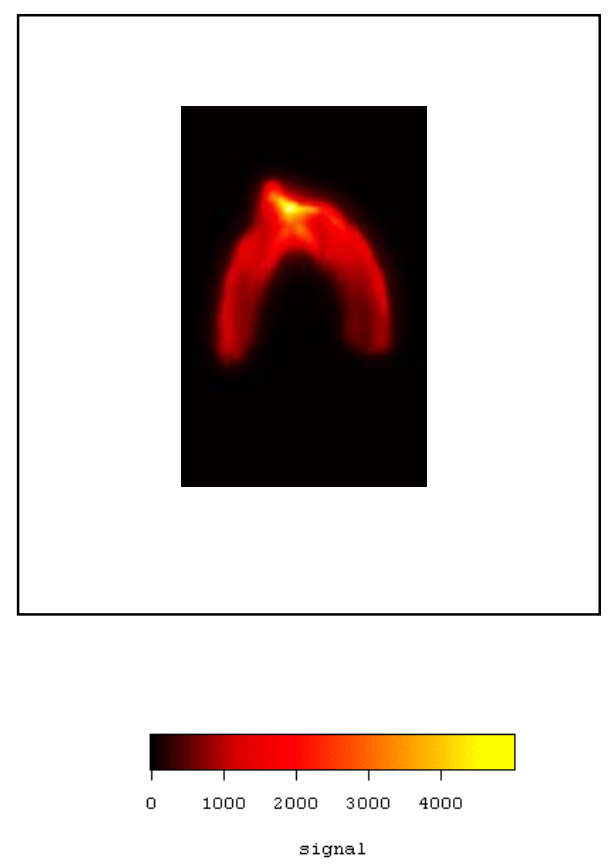

\section{Light signal}
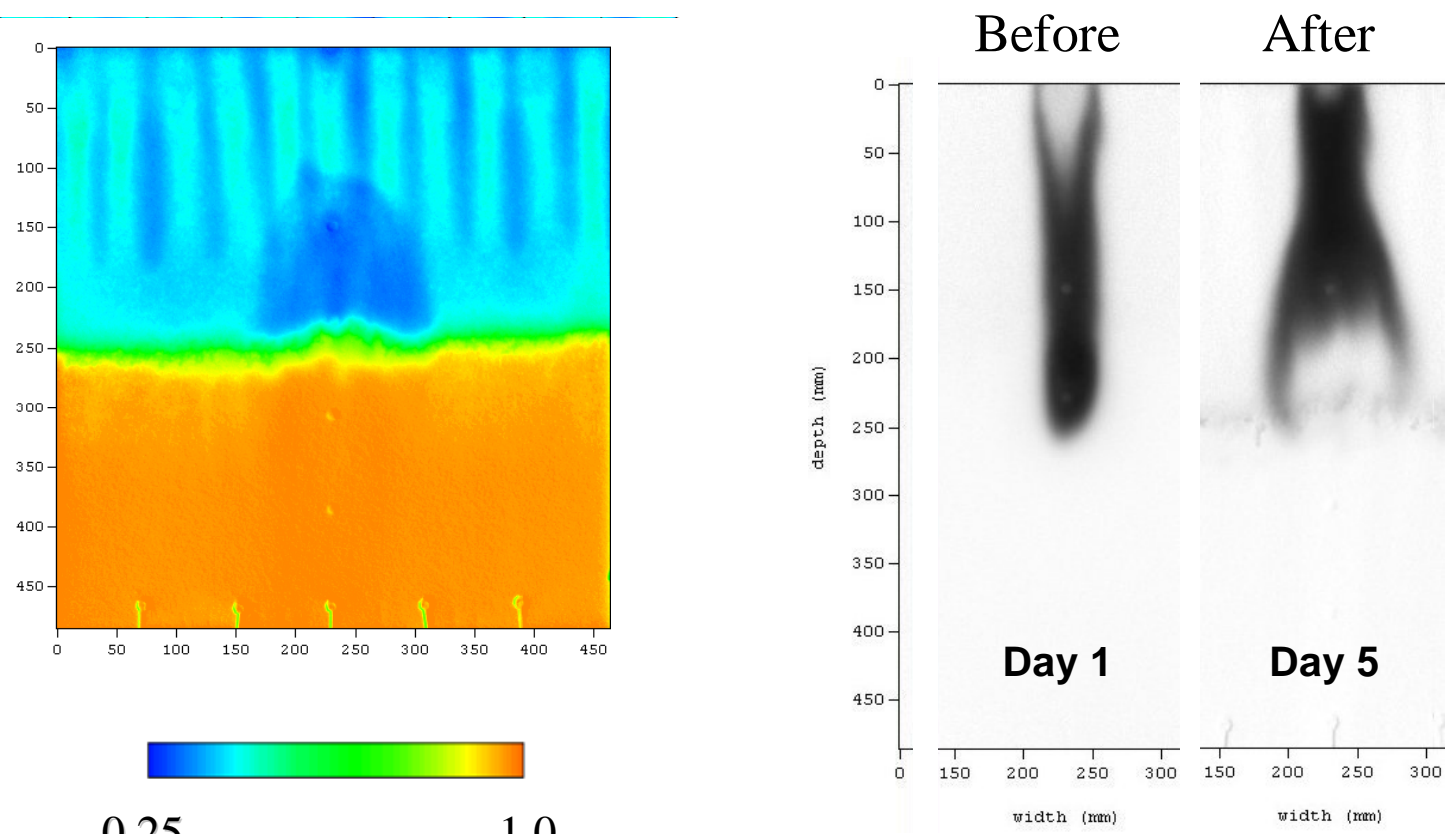

0.25

1.0

Saturation

Figure 2. Direct, Noninvasive Observation of Microbial Distribution and Activity (top) with Simultaneous Monitoring of Water Content Distribution (bottom left) and Flow Paths (bottom right) in Unsaturated Porous Media. Microbial growth resulted in diversion of flow (bottom right) 

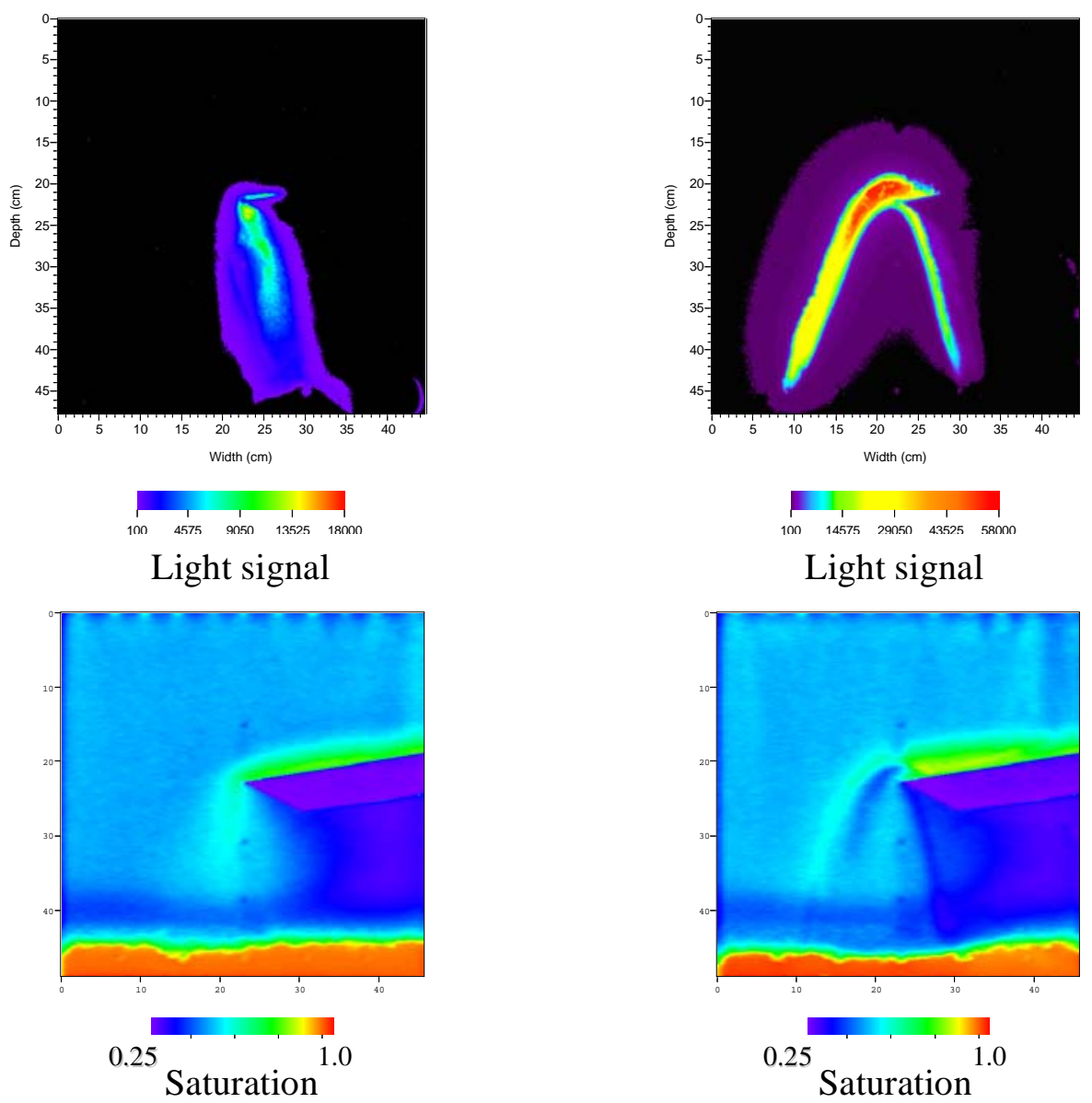

Figure 3. Evolution of Microbial Growth and Activity (top) and Effect of Microbial Growth on Water Distribution (bottom) at Day 2 (left panels) and Day 6 (right panels). Note the scale of the upper right panel is $3 x$ broader than the scale of the upper left panel.

\section{Planned Activities}

Because of the long delay in obtaining samples from the Hanford Site, some project scope definitions have been modified from those of our original proposal:

Samples from the Hanford 216-Z-9 trench site

- Complete analysis of field samples (remainder of FY 2001).

- Evaluate whether isolates from enrichments are useful for unsaturated chamber experiments using gaseous phase nutrients (remainder of FY 2001). 
- Determine effects of starvation, porous media with smaller particle sizes, and natural porous media with broad particle size distributions on motility and colonization (remainder of project).

- Determine to what extent carbon tetrachloride degradation can be achieved at different microbial cell densities in a bulk aerobic system (i.e., the unsaturated zone).

Unsaturated flow chambers

- Develop and use bacterial reporters engineered with constitutive and inducible fluorescent proteins for simultaneous real-time characterization of population density and degradation activity (remainder of FY 2001).

- Continue validation of numerical model for predicting contaminant fate and transport in the vadose zone (remainder of project).

- Investigate the spatial and temporal dynamics of microbial processes using gaseous sources of carbon, nitrogen, and phosphorus (remainder of project). 